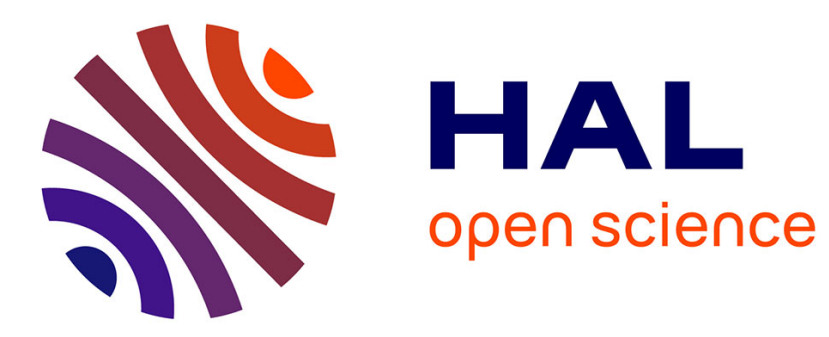

\title{
Stability of spatial queueing systems
}

Charles Bordenave

\section{To cite this version:}

Charles Bordenave. Stability of spatial queueing systems. [Research Report] RR-5305, INRIA. 2004, pp.22. inria-00070695

\section{HAL Id: inria-00070695 \\ https://hal.inria.fr/inria-00070695}

Submitted on 19 May 2006

HAL is a multi-disciplinary open access archive for the deposit and dissemination of scientific research documents, whether they are published or not. The documents may come from teaching and research institutions in France or abroad, or from public or private research centers.
L'archive ouverte pluridisciplinaire HAL, est destinée au dépôt et à la diffusion de documents scientifiques de niveau recherche, publiés ou non, émanant des établissements d'enseignement et de recherche français ou étrangers, des laboratoires publics ou privés. 
INSTITUT NATIONAL DE RECHERCHE EN INFORMATIQUE ET EN AUTOMATIQUE

\title{
Stability of spatial queueing systems
}

\author{
Charles Bordenave*
}

\section{$\mathbf{N}^{\circ} \mathbf{5 3 0 5}$}

Septembre 2004

THÈME 1 



\title{
Stability of spatial queueing systems
}

\author{
Charles Bordenave* \\ Thème 1 - Réseaux et systèmes \\ Projets TREC \\ Rapport de recherche $\mathrm{n}^{\circ} 5305$ - Septembre 2004 † 22 pages
}

\begin{abstract}
In this report, we analyze a queueing system characterized by a space-time arrival process of customers served by a countable set of servers. Customers arrive at some points in space and the server stations have space-dependent processing rates. The workload is seen as a Radon measure and the server stations can adapt their power allocation to the current workload. We derive the stability region of the queuing system in the usual stationary ergodic framework. From the analysis of this stability region, we derive optimal partitions of space among server stations. Some specific subclasses of policies are also studied. Wireless communication networks provides a natural field of application for this model.
\end{abstract}

Key-words: Stability Region, Space-time point process, Wireless networks, Dynamic scheduling.

* INRIA/ENS, 45 rue d'Ulm, 75005 Paris ; e-mail: charles.bordenave@ens.fr 


\section{Stabilité des files d'attente spatiales}

Résumé : Dans ce rapport, nous analysons une file d'attente caractérisée par un processus spacio-temporel d'arrivée de clients servis par un ensemble dénombrable de serveurs. Les clients arrivent sur des points de l'espace et les serveurs ont des vitesses de traitement qui dépendent de l'espace. La quantité de travail est vue comme une mesure de Radon et les serveurs peuvent adapter leur allocation de puissance avec la quantité de travail en cours. Nous établissons la region de stabilité de la file d'attente dans le cadre stationnaire ergodique usuel. L'analyse de la région de stabilité permet de définir des partitions de l'espace entre serveurs optimales. Certaines sous-classes de politiques sont également étudiées. Les réseaux sans fils est le champ d'application naturel de ce model.

Mots-clés : région de stabilité, processus ponctuels spaciaux, réseaux sans fils, politique adaptative. 


\section{Introduction : Model description}

In this paper, we analyze a space-time arrival process of customers served by a countable set of servers. This model is motivated by large scale wireless communications networks but could suit to other types of infinite queuing systems. This model is along the line of other allocation problems studied by Tassiulas and Ephremides [11] and Bambos et al. [8], 1], 3]. Applications of this work to wireless networks are done in [5] .

We consider a system where some jobs arrive exogenously, the jobs are located in the space. Some server stations serve the incoming jobs. Each server station can process the jobs at a rate depending of the localization of the job and a random environment variable.

All the random variables, we introduce in this section are defined on a common probability space $(\Omega, \mathcal{F}, P)$. This space is endowed with a measurable flow $\left\{\theta_{t}\right\}, t \in \mathbb{R}$. We suppose that $\left(P, \theta_{t}\right)$ is ergodic.

\subsection{Customers arrival point process}

In our system, the customers (or jobs) are seen as points of a marked point process $A$. Namely $A$ is a spatial marked point process on $\mathbb{R} \times \mathbb{R}^{d}$ with marks on $\mathbb{R}_{+}$. We use the notation : $A=$ $\sum_{n} \delta_{\left\{T_{n}, X_{n}, \sigma_{n}\right\}}$ to represent the point process. The $\mathrm{n}^{\text {th }}$ job arrives at time $T_{n} \in \mathbb{R}$ located at $X_{n} \in \mathbb{R}^{d}$ and it requires a service time of $\sigma_{n} \in \mathbb{R}_{+}$. We suppose that $A$ is compatible with the flow $\theta$ that is : if $A(\omega)=\sum_{n} \delta_{\left\{T_{n}, X_{n}, \sigma_{n}\right\}}, A \circ \theta_{t}(\omega)=A\left(\theta_{t} \omega\right)=\sum_{n} \delta_{\left\{T_{n}-t, X_{n}, \sigma_{n}\right\}}$. This implies that the point process $A$ is stationary along the first coordinate. The intensity measure of $A$ is defined for a Borel set $B \subset \mathbb{R}^{d+1}$ as :

$$
E(A(B))=E\left(\sum_{n} \mathbb{1}\left(\left(T_{n}, X_{n}\right) \in B\right)\right)
$$

It is supposed to be finite for all compacts $K$. Thus the intensity is a Radon measure of the form : $\lambda(d x) d t$ (see for example Lemma A2.7.11., p.634 in Daley and Vere-Jones [7]). By definition for a compact $K \subset \mathbb{R}^{d+1}$ :

$$
E(A(K))=\int_{K} \lambda(d x) d t<+\infty .
$$

Note that $\lambda$ is not necessarily a finite measure on $\mathbb{R}^{d}$. Therefore between time $t$ and $t^{\prime}>t$, there arrives an infinite number of jobs on the space. In particular, the indexation of customers cannot be time monotonous. However, on a compact set $K \subset \mathbb{R}^{d}$, only a finite number of jobs arrives almost-surely (a.s.).

As usual, we will often drop the " $\omega$ " in the expression of random variables to simplify notations.

Define $P_{A}^{t, x}(\bullet)$ as the Palm probability of the point process $A$ at $(t, x) \in \mathbb{R}^{d+1}$ (refer to 7, chapter 12). Since $A$ is compatible with $\theta_{t}$, we have :

$$
P_{A}^{t, x}(\bullet)=P_{A}^{0, x}\left(\theta_{-t} \bullet\right)
$$

RR $n^{\circ} 5305$ 
Under $P_{A}^{t, x}$, let $\sigma_{t, x}$ be the required service of a customer arriving at time $t$ in $x$. It is consistent since there is a customer arrived at $t$ in $x P_{A}^{t, x}$-a.s.. We will suppose that $0<$ $E^{0, x}\left(\sigma_{0, x}\right)<+\infty, \lambda(d x)$-a.e.. Remind that $E^{0, x}\left(\sigma_{0, x}\right)$ can be understood as the mean number of service time requirement of a typical customer arriving in $x$.

\subsection{Server stations adapative policy}

We have a countable set of server stations denoted by $\mathcal{J}$. The servers can provide service to all points in space and they have a different processing rate for each point in space; the server $j$ serves a customer located at $x$ at a rate of $r_{j}(x)$. We suppose that $x \mapsto r_{j}(x)$ is a bounded positive measurable function and :

$$
\lim _{|x| \rightarrow+\infty} r_{j}(x)=0,
$$

where $|\bullet|$ denotes the Euclidean norm.

The server stations are in a random environment and their processing powers varies over time. At time $t$, the total processing power available is $\epsilon_{j}(t) \in \mathbb{R}_{+}$. As all the other random variables we consider, we suppose that the driving process $\epsilon_{j}(t)$ is compatible with the shift, that is to say : $\epsilon_{j}(t)=\epsilon_{j}(0) \circ \theta_{t}$.

We suppose :

$$
p_{j}=E\left(\epsilon_{j}(0)\right)<+\infty .
$$

The workload at time $t$ is the set of all the jobs waiting to be processed. It is denoted by $W_{t}$ and it is an atomic measure on $\mathbb{R}^{d}$ : for a Borel set $B, \int_{B} W_{t}(d x)$ represents the total service time remaining at time $t$ for all customers located in $B$.

The server stations divide their processing power between the jobs required according to a policy scheme. This power allocation depends on the current workload. We suppose that our queuing system cannot handle an infinite amount of service in finite time for a given location $x$ : more precisely :

$$
\text { for all bounded borel set B, } \quad \sum_{j} p_{j} \sup _{x \in B} r_{j}(x)<+\infty \text {. }
$$

We can define a policy properly.

Definition 1. Let $\mathcal{M}$ be the set of Radon measures on $\mathbb{R}^{d}$, endowed with the vague topology (refer to [7]). A policy is a measurable mapping:

$$
\pi:\left\{\begin{aligned}
\mathcal{M} \times \Omega & \rightarrow \mathcal{M}^{\mathcal{J}} \\
(m, \omega) & \mapsto\left\{\pi_{j}(m, \omega)\right\}, j \in \mathcal{J}
\end{aligned}\right.
$$

satisfying, for all $\omega \in \Omega$ :

$$
\int_{\mathbb{R}^{d}} \pi_{j}(m, \omega)(d x) \leq \epsilon_{j}(0)(\omega)
$$


and

$$
\pi_{j}(m, \omega) \text { is absolutely continuous with respect to } m \text {. }
$$

The policy enforced at time $t$ is : $\pi\left(W_{t}, \theta_{t} \omega\right)$.

Equation (4) means that the server stations cannot allocate more than their total processing power. If the total workload on a Borel set is zero, it is useless to dedicate some processing power to this set. This is the meaning of Condition (15).

$\pi_{j}\left(W_{t}\right)(B)$ is the processing power the server station $j$ dedicates to jobs located in $B$ at time $t$. Therefore, the server station $j$ achieves at time $t$ an instantaneous service rate for a job located in $x$ of :

$$
r_{j}(x) \pi\left(W_{t}\right)(\{x\}) .
$$

The policies we are considering in our model are stochastic and stationary : if $W$ is stationary, that is if $W_{t}=W \circ \theta_{t}$, for a given random measure $W, \pi\left(W_{t}, \theta_{t} \omega\right)=\pi(W, \omega) \circ \theta_{t}$ and thus $\pi$ is stationary. The study of non-stationary policies is performed in [11] in an other framework; in this article the authors show that the non-stationary policies have the same performance as stationary policies.

In the next sections, we will define some interesting classes of policies.

Here is a simple example of a deterministic policy defined for atomic measures :

$$
\pi_{j}^{+}(m)=\left\{\begin{array}{lc}
0 & \text { if }
\end{array} \quad m\right. \text { is the measure zero }
$$

where, $x_{j}^{+}=\arg \max \left\{x: r_{j}(x) \mathbb{1}(m(\{x\})>0)\right\}$. If multiple choices of $x$ are possible, choose the first in the lexicographic order.

With this policy, the server stations serve the user with the best processing rate first. Note in particular that this policy is work conserving: if $W_{t}$ is not the 0 measure, the server is active.

\subsection{Evolution Equation}

The dynamic of our queuing system is given by the following integral equation, for all Borel sets $B$ and $t^{\prime}>t$,

$$
W_{t^{\prime}}(B)=W_{t}(B)+\int_{t}^{t^{\prime}} \int_{B} \sigma_{s, x} A(d s \times d x)-\sum_{j} \int_{t}^{t^{\prime}} \int_{B} r_{j}(x) \pi_{j}\left(W_{s}\right)(d x) d s,
$$

where by definition : $\int_{t}^{t^{\prime}} \int_{B} \sigma_{s, x} A(d s \times d x)=\sum_{n} \sigma_{n} \mathbb{1}\left(T_{n} \in\left[t, t^{\prime}[) \mathbb{1}\left(X_{n} \in B\right)\right.\right.$. If $W_{0}$ is an atomic measure then from Condition (5), $W_{t} \geq 0$ for all $t \geq 0$ and it is also an atomic measure (a.s.).

$\mathrm{RR} \mathrm{n}^{\circ} 5305$ 
Using Borovkov's terminology (see 6], chap. 4), Equation (6) defines a stochastic recursive process : we can write $W_{t+h}=f_{h}\left(W_{t}, A_{t}^{t+h}\right)$, where $A_{t}^{t+h}$ denotes the trajectory of the arrival point process $A$ between $t$ and $t+h$.

Under a given policy $\pi$, for a Radon measure $m$, define $W_{t}^{m}$ as the workload at time $t$ when $W_{0}=m$.

\subsection{Some Examples}

Here come a few examples which illustrate the model.

Example 1. All the jobs arrive on a countable set of points : $\left\{x_{i}\right\}, i \in \mathbb{N}$, with no accumulation points. This points are buffers and Assumption (11) reads :

$$
\lambda_{i}=E\left(A\left([0,1] \times\left\{x_{i}\right\}\right)<+\infty .\right.
$$

The server stations serve users arriving at $x_{i}$ with a processing rate $r_{j}\left(x_{i}\right)$.

In this example, the system reduces to a multi-class job traffic with processing rates depending on the class and the server station. Stability results on this type of systems have been presented in 8 .

Example 2. In a wireless communication scenario, $d=2$ and the server stations are base antennas and the customers are mobile users which want to receive some data from the network. Server station $j$ is located at $Y_{j} \in \mathbb{R}^{2}$. The processing rate can be written : $r_{j}(x)=f(x) L\left(x, Y_{j}\right) . L(x, y)$ is an attenuation function on the channel between $x$ and $y$. A natural assumption is to assume that $L(x, y)$ depends only on $|y-x|$. If customer arrivals are uniform on the plane, then the intensity measure $\lambda(d x)$ is proportional to the Lebesgue measure.

Example 3. When $\epsilon_{j} \in\{0,1\}$, the model has a random connectivity. The server stations are switched on or switched off. At time $t$, if $\epsilon_{j}(t)=1$ (resp. 0) the base station $j$ is switched on (resp. switched off).

\subsection{Stability Region}

In this paper we analyze the stability of the queuing system described above. The definition of stability we use relies on the existence of a stationary regime.

Definition 2. A policy $\pi$ is stable if there exists a finite stationary workload $\left\{M_{t}=M \circ\right.$ $\left.\theta_{t}\right\}, t \in \mathbb{R}$, for an atomic random measure $M$ satisfying Equation (6).

The queuing system is said to be stable if there exists a stable policy.

This definition is called stochastic stability in [3]. In the next section, we will adopt a stronger definition of stability. 
The parameter of the queuing system is the arrival marked point process $A$. Let $\mathcal{N}$ be the set of possible point processes $A$ (namely the point processes of finite intensity and with a service time with finite expectation under the Palm measure). We define :

$$
\begin{aligned}
& \mathcal{F}=\left\{\quad f=\left(x \mapsto f_{j}(x), j \in \mathcal{J}\right)\right. \text { such that, } \\
& \left.\forall j, f_{j} \text { is non negative, measurable and } \lambda(d x) \text {-a.e. } \sum_{j} f_{j}(x)=1\right\} \text {. } \\
& \mathcal{N}^{s}=\left\{A \in \mathcal{N}: \exists f \in \mathcal{F} \text { such that }: \forall j, \int_{\mathbb{R}^{d}} \frac{E_{A}^{0, x}\left(\sigma_{0, x}\right) f_{j}(x)}{r_{j}(x)} \lambda(d x)<p_{j}\right\} . \\
& \overline{\mathcal{N}}^{s}=\left\{A \in \mathcal{N}: \exists f \in \mathcal{F} \text { such that }: \forall j, \int_{\mathbb{R}^{d}} \frac{E_{A}^{0, x}\left(\sigma_{0, x}\right) f_{j}(x)}{r_{j}(x)} \lambda(d x) \leq p_{j}\right\} .
\end{aligned}
$$

These sets are continuous generalizations of the stability sets derived in 8 ].

Note that $\int_{\mathbb{R}^{d}} \frac{E_{A}^{0, x}\left(\sigma_{0, x}\right) f_{j}(x)}{r_{j}(x)} \lambda(d x)$ is a traffic load : $E_{A}^{0, x}\left(\sigma_{0, x}\right) \lambda(d x)$ is the mean number of service requirements per unit of surface and $r_{j}(x)$ is the processing rate at $x$ for server station $j$.

We can now state the following stability theorem :

Theorem 1. For the queuing model described above,

- if $A \in \mathcal{N}^{s}$, then there exists a stable policy,

- if there is a stable policy then $A \in \overline{\mathcal{N}}^{s}$.

Note that, as in Loynes' Theorem, the stability region depends on the distribution of the point process $A$ only through its means. To fix ideas, suppose that there is only one server station and that all jobs arrive at the same place, say 0 . Then the theorem says that the stability region is given by $E_{A}^{0,0}\left(\sigma_{0,0}\right) \lambda(0)<p_{1} r_{1}(0)$. This result is the usual condition $\rho<1$ for $\mathrm{G} / \mathrm{G} / 1$ queues.

In the proof of the theorem, we establish that to a given stable policy $\pi$ corresponds a set of functions $\left(f_{j}\right)_{j \in \mathcal{J}}$ in $\mathcal{F}$ such that :

$$
\int_{\mathbb{R}^{d}} \frac{E_{A}^{0, x}\left(\sigma_{0, x}\right) f_{j}(x)}{r_{j}(x)} \lambda(d x) \leq p_{j}
$$

$f_{j}(x)$ is the proportion of service carried by the server $j$ for users in $x$ in the stationary regime and for the stable policy $\pi$.

The converse mapping is also available, for a set of functions $\left(f_{j}\right)_{j \in \mathcal{J}}$ in $\mathcal{F}$ such that the above inequality is satisfied with a strict inequality, there exists a stable policy. In fact, as it will be seen in the proof of Theorem 1 this last assertion is only true for a dense subset of functions of $\mathcal{F}$.

A proof of Theorem 1 is given in Section 2

$\mathrm{RR} \mathrm{n}^{\circ} 5305$ 
In Section [3 we give a stronger stability results on our queuing systems which follows from classical coupling arguments.

In Section 4 we consider a subclass of policies which preserves a kind of monotony for the workload measure.

Section [5 is dedicated to the stability region defined by Equation (7) when the spatial intensity measure in absolutely continuous with respect to the Lebesgue measure.

The last results of this paper concern a subclass of policy for which the stability region is easy to compute. This work is done in Section [

\section{Proof of Theorem 1}

\subsection{Necessary Condition}

This technical lemma is needed in what follows.

Lemma 1. Let $Z$ be a non-negative a.s. finite random variable, such that for a given $t$, $Z-Z \circ \theta_{t} \in L^{1}(P)$, then $E\left(Z-Z \circ \theta_{t}\right)=0$.

For the proof, see for example Lemma 2.2.1 of Baccelli and Bremaud [2].

Suppose exists a stable policy $\pi$. There exists a stationary workload measure $W_{t}=W \circ \theta_{t}$ a.s. finite. Let $B$ be a bounded Borel set, from Equation ([6), we have :

$$
\left.W \circ \theta_{t}(B)=W(B)+\int_{0}^{t} \int_{B} \sigma_{s, x} A(d s \times d x)-\sum_{j} \int_{t}^{t+h} \int_{B} r_{j}(x) \pi_{j}\left(W \circ \theta_{s}\right)\right)(d x) d s .
$$

The Campbell Formula for marked point processes, using (1.1), implies :

$$
\begin{aligned}
E\left(\int_{0}^{t} \int_{B} \sigma_{s, x} A(d s \times d x)\right) & =\int_{0}^{t} \int_{B} \int_{\mathbb{R}_{-}} \sigma_{s, x} P_{A}^{s, x}(d \sigma) \lambda(d x) d s \\
& =t \int_{B} E_{A}^{0, x}\left(\sigma_{0, x}\right) \lambda(d x) .
\end{aligned}
$$

Since $\pi_{j}(B)$ is bounded by $\epsilon_{j}$ and we can define $\bar{\pi}_{j}(B)=E\left(\pi_{j}(W)(B)\right) . \bar{\pi}_{j}$ is a Radon measure. Moreover, since $\int_{\mathbb{R}^{d}} \pi_{j}(W)(d x) \leq \epsilon_{j}$, we have : $\int_{\mathbb{R}^{d}} \bar{\pi}_{j}(d x) \leq p_{j}$. Using Condition (3), we deduce :

$$
E\left(\left|W \circ \theta_{t}(B)-W(B)\right|\right) \leq t \int_{B} E_{A}^{0, x}\left(\sigma_{0, x}\right) \lambda(d x)+t \sum_{j} p_{j} \sup _{x \in B} r_{j}(x)<+\infty .
$$

Thus $W \circ \theta_{t}(B)-W(B) \in L^{1}(P)$ and we can apply Lemma 1 to conclude that :

$$
0=\int_{B} E_{A}^{0, x}\left(\sigma_{0, x}\right) \lambda(d x)-\sum_{j} \int_{B} r_{j}(x) \bar{\pi}_{j}(d x) .
$$


Since Equation (9) holds for all bounded Borel sets, the Radon measures : $\sum_{j} r_{j}(x) \bar{\pi}_{j}(d x)$ and $E_{A}^{0, x}\left(\sigma_{0, x}\right) \lambda(d x)$ are equal. In particular the measure $r_{j}(x) \bar{\pi}_{j}(d x)$ is absolutely continuous with respect to $E_{A}^{0, x}\left(\sigma_{0, x}\right) \lambda(d x)$. We define the measurable function $\tilde{\pi}_{j}(x)$ as the Radon-Nicodym derivative of $\bar{\pi}_{j}(d x)$ with respect to $\lambda(d x)$. Let $f_{j}(x)=\frac{r_{j}(x) \tilde{\pi}_{j}(x)}{E_{A}^{0, x}\left(\sigma_{0, x}\right)}$. We deduce from (9) that for all Borel $B$ :

$$
\int_{B} E_{A}^{0, x}\left(\sigma_{0, x}\right) \lambda(d x)=\sum_{j} \int_{B} f_{j}(x) E_{A}^{0, x}\left(\sigma_{0, x}\right) \lambda(d x) .
$$

Thus $\lambda(d x)$-a.e. : $\sum_{j} f_{j}(x)=1$.

We have proved that $f \in \mathcal{F} . \int_{\mathbb{R}^{d}} \bar{\pi}_{j}(d x) \leq p_{j}$ reads $\int_{\mathbb{R}^{d}} \frac{E_{A}^{0, x}\left(\sigma_{0, x}\right) f_{j}(x)}{r_{j}(x)} \lambda(d x) \leq p_{j}$.

The second assertion of Theorem 1 follows.

\subsection{Sufficient Condition}

Suppose, $A \in \mathcal{N}^{s}$. There exists $\left(f_{j}\right), j \in \mathcal{J}$, in $\mathcal{F}$ such that : for all $j, \int_{\mathbb{R}^{d}} \frac{E_{A}^{0, x}\left(\sigma_{0, x}\right) f_{j}(x)}{r_{j}(x)} \lambda(d x)=$ $\rho_{j}<1$. We can suppose that $f$ has the properties given in Proposition 6 (in Appendix). For a given policy $\pi$ and an initial atomic workload $m$, we define the sets :

$$
\mathcal{A}_{j}^{m}(t)=\left\{x: r_{j}(x) \int_{0}^{t} \pi_{j}\left(W_{s}^{m}\right)(\{x\}) d s \leq f_{j}(x)\left(m(\{x\})+\int_{[0, t) \times\{x\}} \sigma_{s, x} A(d s \times d x)\right)\right\} .
$$

The fact that $x$ is in $\mathcal{A}_{j}^{m}(t)$ means that server $j$ has contributed for less than $f_{j}(x)$ to fulfill the service requirements of customers located in $x$.

Consider the following non-stationary policy : for all $j \in \mathcal{J}$, for $t \geq 0$ :

$$
\pi_{j}:\left\{\begin{aligned}
\Omega \times \mathbb{R} & \rightarrow \mathcal{M} \\
(\omega, t) & \mapsto\left\{\begin{array}{lll}
\epsilon_{j}(t) \delta_{x_{j}^{*}} & \text { if } & \mathcal{A}_{j}^{W_{0}}(t) \neq \emptyset \\
0 & \text { if } & \mathcal{A}_{j}^{W_{0}}(t)=\emptyset .
\end{array}\right.
\end{aligned}\right.
$$

where,

$$
\left.x_{j}^{*}=\arg \max \left\{x: \mathbb{1}\left(x \in \mathcal{A}_{j}^{W_{0}}(t)\right) \mathbb{1}\left(W_{t}(\{x\})>0\right)\right) r_{j}(x)\right\}
$$

if multiple choices of $x_{j}^{*}$ are possible, choose the first in the lexicographic order.

The existence of this policy follows from Condition (2).

The policy $\pi$ divides the workload among all servers with proportions $f_{j}$ and processes the jobs at the faster available rate. This policy is not stationary and is defined for an initial workload at time 0 . In other words, $\pi_{j}(t)$ is the policy enforced at time $t$ when at time 0 , the workload was equal to $m$ : with the usual notation, $\pi_{j}(t)=\pi_{j}\left(W_{t}^{m}\right)$. 
Note also that if at time $T, \mathcal{A}_{j}^{m}(T)=\emptyset$, then the server $j$ will only serve after time $T$ jobs arrived after time $T$ and for $t>0$ :

$$
\mathcal{A}_{j}^{m}(t+T)=\mathcal{A}_{j}^{0}(t) \circ \theta_{T} .
$$

Let $B$ be a bounded Borel set, we have :

$$
\begin{aligned}
W_{t}(B) & =\int_{0}^{t} \int_{B} \sigma_{s, x} A(d s \times d x)-\sum_{j} \int_{0}^{t} \int_{B} r_{j}(x) \pi_{j}(s)(d x) d s \\
& =\sum_{j} \int_{0}^{t} \int_{B} f_{j}(x) \sigma_{s, x} A(d s \times d x)-\sum_{j} \int_{0}^{t} \int_{B} r_{j}(x) \pi_{j}(s)(d x) d s \\
& =\sum_{j} \int_{B} r_{j}(x) \tilde{W}_{t}^{j}(d x),
\end{aligned}
$$

where :

$$
\tilde{W}_{t}^{j}=\int_{0}^{t} \int_{B} \frac{f_{j}(x) \sigma_{s, x}}{r_{j}(x)} A(d s \times d x)-\int_{0}^{t} \int_{B} \pi_{j}(s)(d x) d s .
$$

Definition 3. We define a total order $\prec_{j}$ on $\mathbb{R}^{d}: x \succ_{j} y$ if $r_{j}(x)>r_{j}(y)$ or $r_{j}(x)=r_{j}(y)$ and $x$ is in the lexicographic order smaller than $y$.

$A$ Borel set $B$ is a j-max set if : $\left\{x: x \succeq_{j}\right.$ y for all $\left.y \in B\right\}=B$.

Lemma 2. For a j-max set $B$ :

$$
\begin{aligned}
\tilde{W}_{t}^{j}(B)=(\max \quad & \left(\int_{B} \frac{f_{j}(x) W_{0}(d x)}{r_{j}(x)}+\int_{0}^{t} \int_{B} \frac{f_{j}(x) \sigma_{s, x}}{r_{j}(x)} A(d s \times d x)-\int_{0}^{t} \epsilon_{j}(s) d s,\right. \\
& \left.\left.\sup _{0 \leq h \leq t} \int_{0}^{h} \int_{B} \frac{f_{j}(x) \sigma_{s, x}}{r_{j}(x)} A(d s \times d x)-\int_{0}^{h} \epsilon_{j}(s) d s\right)\right)^{+} .
\end{aligned}
$$

Proof. The policy $\pi$ divides the global queuing system with the set of servers $\mathcal{J}$ into distinct queues, one for each server in $\mathcal{J}$ with an incoming workload equal to :

$$
\sum_{j} \int_{-t}^{0} \int_{B} f_{j}(x) \sigma_{s, x} A(d s \times d x) .
$$

$\tilde{W}_{t}^{j}(B)$ is the rescaled workload : the server $j$ serves the user at $x$ with a unit processing rate. If $B$ is a j-max set, from the definition of policy $\pi_{j}$, the server $j$ dedicates all of its processing power to $B$ if the workload in $\mathrm{B}$ is not 0 . The customers in $\mathbb{R}^{d} \backslash B$ are served if there is no customer in $B$. Thus the statement of the lemma follows from the usual formula for the $\mathrm{G} / \mathrm{G} / 1$ queue. 
Let $M_{t}=W_{t}^{0} \circ \theta_{-t}$ be the Loynes sequence under policy $\pi$ and $\tilde{M}_{t}^{j}=\tilde{W}_{t}^{0} \circ \theta_{-t}$.

$$
M_{t}(B)=\sum_{j} \int_{B} r_{j}(x) \tilde{M}_{t}^{j}(d x)
$$

. Note from Lemma 2 that $\tilde{M}_{t}^{j}(B)$ is a non-decreasing sequence for a $j$-max set.

Lemma 3. As t tends to infinity, $\tilde{M}_{t}^{j}$ couples a.s. with $\tilde{M}_{\infty}^{j}$, a finite random measure.

Proof. Let $B$ be a j-max set, from the Birkhoff's Theorem a.s. :

$$
\lim _{t \rightarrow+\infty} \frac{1}{t} \int_{-t}^{0} \int_{B} \frac{f_{j}(x) \sigma_{s, x}}{r_{j}(x)} A(d s \times d x)=\int_{B} \frac{f_{j}(x) E_{A}^{0, x}\left(\sigma_{0, x}\right)}{r_{j}(x)} \lambda(d x)<1 .
$$

Therefore : $\tilde{M}_{t}^{j}(B)=\left(\sup _{0 \leq h \leq t} \int_{-h}^{0} \int_{B} \frac{f_{j}(x) \sigma_{s, x}}{r_{j}(x)} A(d s \times d x)-h\right)^{+}$is a bounded increasing sequence and it couples with $\tilde{M}_{\infty}^{j}(B)=\left(\sup _{h \in \mathbb{R}_{+}} \int_{-h}^{0} \int_{B} \frac{f_{j}(x) \sigma_{s, x}}{r_{j}(x)} A(d s \times d x)-h\right)^{+}$.

It remains to prove the result for any Borel set. $\mathbb{R}^{d}$ is a $j$-max set and we have for any set $B: \tilde{M}_{t}^{j}(B) \leq \tilde{M}_{t}^{j}\left(\mathbb{R}^{d}\right) \leq \tilde{M}_{\infty}^{j}\left(\mathbb{R}^{d}\right)$. Therefore a subsequence $\tilde{M}_{t_{n}}^{j}(B)$ converges to $\tilde{M}_{\infty}^{j}(B)$ and $\tilde{M}_{\infty}^{j}$ is well defined and it is a finite random measure.

Since $\tilde{W}_{t}^{j}\left(\mathbb{R}^{d}\right)$ is a $\mathrm{G} / \mathrm{G} / 1$ queue, we can define $T$ the first time after the coupling time with $\tilde{M}_{\infty}^{j} \circ \theta_{t}\left(\mathbb{R}^{d}\right)$ such that $\tilde{W}_{t}^{j}\left(\mathbb{R}^{d}\right)=0$. $T$ is a.s. finite. In view of Equation (11), for $t \geq T, \tilde{W}_{t}^{j}=\tilde{M}_{\infty}^{j} \circ \theta_{t}$. Thus : $\tilde{M}_{t}^{j}=\tilde{W}_{t}^{j} \circ \theta_{-t}=\tilde{M}_{\infty}^{j}$ and $\tilde{M}_{t}^{j}$ couples with $\tilde{M}_{\infty}^{j}$.

Let $B$ be a bounded set. For our specifical choice of $f$, given by Proposition [6]:

$$
M_{t}(B)=\sum_{j \in \mathcal{J}_{B}} \int_{B} r_{j}(x) \tilde{M}_{t}^{j}(d x),
$$

Since $\left|\mathcal{J}_{B}\right|$ is finite, we deduce from Lemma 3 that $M_{t}(B)$ couples a.s. with $M_{\infty}(B)=$ $\sum_{j \in \mathcal{J}_{B}} \int_{B} r_{j}(x) \tilde{M}_{\infty}^{j}(d x)$.

We thus have proved the existence of the limit :

$$
\lim _{t \rightarrow \infty} M_{t}=M_{\infty}=\sum_{j} \int_{B} r_{j}(x) \tilde{M}_{\infty}^{j}(d x) .
$$

To conclude the proof, it remains to prove that $M_{\infty}$ is a stationary solution of Equation (6) for a policy $\pi^{\prime}$.

Along the line of Lemma 3 we can also prove that the process $\left\{\tilde{M}_{t+s}^{j} \circ \theta_{s}\right\}_{0 \leq s \leq h}$ couples with $\left\{\tilde{M}_{\infty}^{j} \circ \theta_{s}\right\}_{0 \leq s \leq h}$ for any positive $h$.

From Equation (12), for a bounded set $B$ :

$$
\begin{aligned}
\tilde{M}_{t+h}^{j} \circ \theta_{h}(B) & =\int_{-t-h}^{0} \int_{B} \frac{f_{j}(x) \sigma_{s, x}}{r_{j}(x)} A(d s \times d x)-\int_{0}^{t+h} \int_{B} \pi_{j}\left(\theta_{-t}, s\right)(d x) d s \\
& =\tilde{M}_{t}^{j}(B)+\int_{0}^{h} \int_{B} \frac{f_{j}(x) \sigma_{s, x}}{r_{j}(x)} A(d s \times d x)-\int_{0}^{h} \int_{B} \pi_{j}\left(\theta_{-t}, t+s\right)(d x) d s .
\end{aligned}
$$

$\mathrm{RR} \mathrm{n}^{\circ} 5305$ 
If $Z$ is the maximum of coupling time of $\left\{\tilde{M}_{t+s}^{j} \circ \theta_{s}(B)\right\}_{0 \leq s \leq h}$, for $t \geq Z$ we deduce :

$$
\int_{0}^{h} \int_{B} \pi_{j}\left(\theta_{-t}, t+s\right)(d x) d s=\tilde{M}_{\infty}^{j} \circ \theta_{h}(B)-\tilde{M}_{\infty}^{j}(B)+\int_{0}^{h} \int_{B} \frac{f_{j}(x) \sigma_{s, x}}{r_{j}(x)} A(d s \times d x) .
$$

For all $h$, the right hand side of the last equation does not depend on $t$, thus $\pi_{j}\left(\theta_{-t}, t+s\right)$ couples a.e. with a measure $\pi_{j}^{\prime}(s)=\lim _{t} \pi_{j}\left(\theta_{-t}, t+s\right)$. Let $t_{0}$ such that the coupling occurs, we have $\pi_{j}^{\prime}(s)=\lim _{t} \pi_{j}\left(\theta_{-t+s-t_{0}} \circ \theta_{s}, t+t_{0}-s+s\right)=\pi_{j}^{\prime}\left(t_{0}\right) \circ \theta_{s-t_{0}}$.

In consequence, $\pi^{\prime}$ is a stationary policy and $M_{\infty}$ is a stationary solution of Equation (6).

Theorem 1 is proved.

Remark 1. In the particular case described in Example 1, a simpler proof is available. We have : $\sum_{i} \frac{\lambda_{i} E_{A}^{0, x_{i}}\left(\sigma_{0, x_{i}}\right) f_{j}\left(x_{i}\right)}{r_{j}\left(x_{i}\right)}=\rho_{j}<1$. Consider the following deterministic policy defined for atomic measure with atoms on $\left\{x_{i}\right\}, i \in \mathbb{N}$ :

$$
\pi_{j}(m)\left(\left\{x_{i}\right\}\right)=\epsilon_{j} \mathbb{1}\left(m\left(\left\{x_{i}\right\}\right) \neq 0\right) \frac{f_{j}\left(x_{i}\right) \lambda_{i} E_{A}^{0, x_{i}}\left(\sigma_{0, x_{i}}\right)}{r_{j}\left(x_{i}\right) \rho_{j}} .
$$

When computing $M_{t}\left(\left\{x_{i}\right\}\right)$, it appears that this policy is stable.

\section{Convergence toward a Stationary Solution}

When there exists a stationary regime in a queuing system it is an important issue to know if for any initial condition the workload converges in some sense to the stationary regime. The following proposition gives a positive answer for the policy defined in the proof of Theorem 11

Proposition 1. If the policy scheme defined by Equation (10) is enforced, for any finite initial workload at time $t=0$, for all bounded Borel set $B,\left\{W_{t+T}(B)\right\}, t \in \mathbb{R}_{+}$, converges in variation toward $\left\{M \circ \theta_{t}(B)\right\}, t \in \mathbb{R}_{+}$, as $T$ tends to infinity.

Note that the workload measure does not converge in variation, convergence happens only on bounded sets. The proposition states that the workload converges in variation for the vague topology.

Proof. The proof relies on the following fact : if a stochastic process $\left\{X_{t}\right\}$ couples with $\left\{Y \circ \theta_{t}\right\}$ then $\left\{X_{t+T}\right\}, t \in \mathbb{R}_{+}$, converges in variation toward $\left\{Y \circ \theta_{t}\right\}, t \in \mathbb{R}_{+}$, as $T$ tends to infinity (see Lindvall [9] or Theorem 2.4.1. of [2]).

From Lemma 2] $\tilde{W}_{t}^{j}(B)$ is $\mathrm{G} / \mathrm{G} / 1$ queue, therefore the coupling of $\tilde{W}_{t}^{j}(B)$ for any initial condition follows from Property 2.4.1. in 2].

For a general Borel set $B$, it suffices to notice as already done that $\tilde{W}_{t}^{j}(B) \leq \tilde{W}_{t}^{j}\left(\mathbb{R}^{d}\right)$. The same arguments used in the proof of Theorem 1 works to show that $\tilde{W}_{t}^{j}(B)$ couples for any initial condition.

If $B$ is a bounded set, $W_{t}(B)=\sum_{j \in \mathcal{J}_{B}} \int_{B} r_{j}(x) \tilde{M}_{t}^{j}(d x)$. Since $\left|\mathcal{J}_{B}\right|$ is finite, the coupling also occurs. 


\section{Monotonous Policies}

The policy we have defined to prove the sufficiency part of Theorem 1 is not of particular interest. In particular, it requires the knowledge of the mappings $f_{j}(x)$. Along the line of the work done in [1], 1], 3], it would be very appealing to find some stable policy which does not rely on the knowledge of the parameters of the system.

\subsection{Loynes' Sequence and Loynes' Variable}

We are now going to use the key ideas of the proof of Loynes for general Polish space (see paragraph 2.5.2. of [2]). The space $\mathcal{M}$ is a Polish space for the vague topology (refer to [7]).

We define a partial order $\preceq$ on $\mathcal{M}$, the set of Radon measures on $\mathbb{R}^{d}$. Let $m$ and $m^{\prime}$ be two Radon measures, $m \preceq m^{\prime}$ if for all bounded Borel set $B$ in $\mathbb{R}^{d}, m(B) \leq m^{\prime}(B)$.

Lemma 4. Let $\left(m_{n}, n \in \mathbb{N}\right)$ be a monotonous sequence in $\mathcal{M}$ for $\preceq$, such that for all bounded Borel sets $B, m_{n}(B)$ is bounded. Then, $\left(m_{n}, n \in \mathbb{N}\right)$ converges in $\mathcal{M}$ for the vague topology.

Proof. Let $f$ be a bounded continuous function vanishing outside a bounded set. Let $f_{+}=$ $\max (0, f)$ and $f_{-}=\min (0, f)$, it is straightforward to check that $\left(m_{n}\left(f_{+}\right), n \in \mathbb{N}\right)$ and $\left(m_{n}\left(f_{-}\right), n \in \mathbb{N}\right)$ are bounded monotonous sequences. Thus $\left(m_{n}(f), n \in \mathbb{N}\right)$ converges.

The Loynes sequence $\left(M_{t}, t \in \mathbb{R}_{+}\right)$is defined as the workload found at time 0 supposing that the workload was zero at time $-t$, that is :

$$
M_{t}=W_{t}^{0} \circ \theta_{-t} .
$$

The Loynes sequence is of particular interest for the class of monotonous policies.

A policy is said to be monotonous if $m^{\prime} \preceq m$ implies for all $t \in \mathbb{R}_{+}, W_{t}^{m^{\prime}} \preceq W_{t}^{m}$. Monotonous policies are quite natural in our queuing setting.

Lemma 5. Suppose $\pi$ is a monotonous policy then $\left\{M_{t}\right\}, t \in \mathbb{R}_{+}$, is non-increasing sequence (for $\preceq$ ).

Proof. Let $h \in \mathbb{R}_{+}$, we have : $0=W_{0}^{0} \circ \theta_{-t} \preceq W_{h}^{0} \circ \theta_{-t-h}$, from the monotonicity of $m \mapsto W_{t}^{m}$, we deduce that : $M_{t}=W_{t}^{0} \circ \theta_{-t} \preceq W_{t}^{W_{h}^{0} \circ \theta_{-t-h}} \circ \theta_{-t}=M_{t+h}$.

If $\left(M_{t}\right), t \in \mathbb{R}_{-}$, is an non-decreasing sequence and is bounded by a random Radon measure $Z$ (for $\preceq$ ), using Lemma $4 M_{t}$ converges a.s. in $\mathcal{M}$ and we can then define the Loynes variable as :

$$
M_{\infty}=\lim _{t \rightarrow+\infty} M_{t}
$$

$\mathrm{RR} \mathrm{n}^{\circ} 5305$ 


\subsection{Monotonous Left Continuous Policies}

In this paragraph, we gives some conditions to ensure that a stationary solution exists. To ensure that the Loynes' variable is a stationary solution we need some continuity properties of $\pi_{j}$.

Definition 4. Let $f$ be a measurable mapping from $\mathcal{M}$ to $\mathcal{M}$. We say that $f$ is left continuous (resp. right continuous) if for all non-decreasing (resp. non-increasing) converging sequence $\left(m_{n}\right), n \in \mathbb{N}$, of $\mathcal{M}, \lim _{n} f\left(m_{n}\right)=f\left(\lim _{n} m_{n}\right)$.

It is consistent to define some continuity properties with converging sequences since $\mathcal{M}$ is a complete metric space. Note also that a mapping can be left and right continuous without being continuous. This is due to the fact that $\preceq$ is only a partial order.

Right continuous policies cannot be relevant. When a new customer arrives, the policy can change drastically. In particular remark that a work-conserving policy cannot be right continuous.

We define the discontinuity set of a mapping $h: \operatorname{disc}(f)$ as $\{x: h(x)$ not continuous at $x\}$.

Proposition 2. Suppose $\pi_{j}$ is left continuous and $\lambda\left(\operatorname{disc}\left(r_{j}\right)\right)=0$ for all $j \in \mathcal{J}$. When $M_{\infty}$ is finite, it is a stationary solution of Equation (6).

Proof. By definition, $W_{s}^{M_{t}}=W_{t+s}^{0} \circ \theta_{-t}=W_{t+s}^{0} \circ \theta_{-s-t} \circ \theta_{s}=M_{t+s} \circ \theta_{s}$. Therefore, from Equation (6), we have, for a Borel set $B$ and $t \in \mathbb{R}^{+}$:

$$
\begin{aligned}
M_{t+h} \circ \theta_{h}(B)= & W_{h}^{M_{t}}(B) \\
= & M_{t}(B)+\int_{0}^{h} \int_{B} \sigma_{s, x} A(d s, d x) \\
& \quad-\sum_{j} \int_{0}^{h} \int_{B} r_{j}(x) \pi_{j}\left(M_{t+s} \circ \theta_{s}\right)(d x) d s .
\end{aligned}
$$

When $\left(t_{k}\right), k \in \mathbb{N}$, be an increasing sequence converging toward $+\infty$. $\left(M_{t_{k}+s} \circ \theta_{s}\right), k \in \mathbb{N}$, is a non-decreasing sequence converging toward $M_{\infty} \circ \theta_{s}$. Since $\pi_{j}$ is left continuous, we have $\lim _{k \rightarrow+\infty} \pi_{j}\left(M_{t_{k}+s} \circ \theta_{s}\right)=\pi_{j}\left(M_{\infty} \circ \theta_{s}\right)$ for the vague convergence.

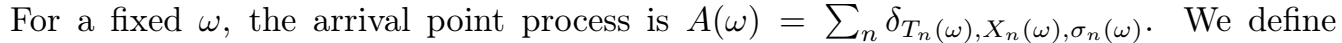
$C(\omega)=\left\{X_{n}(\omega), n \in \mathbb{N}\right\}$ and let $B$ be a bounded Borel set such that $C(\omega) \cap \partial B=\emptyset$. $\partial B$ avoids a countable set of points in $\mathbb{R}^{d}$.

From Equation (6) and Condition (5), $M_{\infty} \circ \theta_{s}$ and $\pi_{j}\left(M_{\infty}\right) \circ \theta_{s}$ are atomic measures and they are supported by $\sum_{k} \delta_{X_{k}} \mathbb{1}\left(T_{k}<s\right)$. Thus, for $B$ as above, for all $s$ in $\mathbb{R}$, we have $\pi_{j}\left(M_{\infty} \circ \theta_{s}\right)(\partial B)=0$. Moreover, since $\lambda\left(\operatorname{disc}\left(r_{j}\right)\right)=0, P$-almost surely $\pi\left(M_{\infty} \circ\right.$ $\left.\theta_{s}\right)\left(\operatorname{disc}\left(r_{j}\right)\right)=0$. From Lemma 6] (in Appendix), we deduce $P$-almost surely :

$$
\lim _{k \rightarrow+\infty} \int_{B} r_{j}(x) \pi_{j}\left(M_{t_{k}+s} \circ \theta_{s}\right)(d x)=\int_{B} r_{j}(x) \pi_{j}\left(M_{\infty} \circ \theta_{s}\right)(d x) .
$$


Now, from Hypothesis (3), $\sum_{j} \int_{0}^{h} \int_{B} r_{j}(x) \pi_{j}\left(M_{t+s} \circ \theta_{s}\right)(d x) \leq \sum_{j} h \sup _{x \in B} r_{j}(x)<+\infty$. The dominated convergence theorem gives, $P$-almost surely :

$$
M_{\infty} \circ \theta_{h}(B)=M_{\infty}(B)+\int_{0}^{h} \int_{B} \sigma_{s, x} A(d s, d x)-\sum_{j} \int_{0}^{h} \int_{B} r_{j}(x) \pi_{j}\left(M_{\infty} \circ \theta_{s}\right)(d x) .
$$

From Lemma 7 (in Appendix), this last equation is indeed satisfied for all Borel sets. Therefore, $M_{\infty}$ is a stationary solution.

The hypothesis can be made more precise. Consider $E=\left\{\omega \in \Omega: \exists T, \forall t>T M_{t}(\omega)=\right.$ $\left.M_{\infty}(\omega)\right\}$. On $E, M_{t}$ converges in variation (or couples).

We have already noticed that : $M_{t+s}\left(\theta_{s} \omega\right)=W_{s}^{M_{t}}(\omega)$. Thus, if $M_{t}(\omega)=M_{\infty}(\omega)$, for $t>T$, we deduce that $M_{t}\left(\theta_{s} \omega\right)=W_{s}^{M_{t-s}}(\omega)=M_{\infty}\left(\theta_{s} \omega\right)$ for $t>T+s$. Thus $E$ is a $\theta_{s}$-invariant event and from ergodicity, $P(E) \in\{0,1\}$.

If $P(E)=1, M_{t}$ couples with $M_{\infty}$ a.s.. It follows that the hypothesis on the continuity of $\pi_{j}$ and $r_{j}$ are not needed. We deduce the corollary :

Corollary 1. For a given policy $\pi$, if $M_{t}=W_{t}^{Z} \circ \theta_{-t}$ couples with $M_{\infty}$ then $M_{\infty}$ is a stationary workload solution of Equation (6).

Example 4. Cone policies. An interesting class of policies has emerged in papers, see [1], 1], [3. Let $\alpha>0$, we define for an atomic policy $m$ :

$$
\begin{gathered}
A_{j}(m)=\arg \max \left\{x: m(\{x\})^{\alpha} r_{j}(x)\right\} \\
\pi_{j}(m)=\left\{\begin{array}{lll}
C(m) \epsilon_{j} \sum_{x \in A_{j}(m)} r_{j}(x)^{-\frac{\alpha+1}{\alpha}} \delta_{x} & \text { if } & m \neq 0 \\
0 & \text { if } & m=0,
\end{array}\right.
\end{gathered}
$$

where $C(m)$ is the constant such that $\int_{\mathbb{R}^{d}} \pi_{j}(m)(d x)=\epsilon_{j}$ if $m \neq 0$.

Notice that $\pi(\mathrm{cm})=\pi(m)$ for $c>0$. For finite workload measures, it can be shown that this policy is monotonous and left-continuous. However, it is not clear whether or not this policy is stable when $A \in \mathcal{N}^{s}$.

\section{Optimal Spatial Allocation}

In this section, we suppose that the spatial arrival intensity $\lambda(d x)$ is absolutely continuous with respect to the Lebesgue measure : it can be written as $\lambda(x) d x$.

We have seen in Theorem 1 that the stability of system relies on :

$$
\rho=\inf _{f \in \mathcal{F}} \sup _{j \in \mathcal{J}} \frac{1}{p_{j}} \int_{\mathbb{R}^{d}} \frac{E_{A}^{0, x}\left(\sigma_{0, x}\right) f_{j}(x)}{r_{j}(x)} \lambda(x) d x .
$$


If $\rho<1$, the system is stable, if $\rho>1$, the system is unstable. In this section, we analyse this optimization problem. To this end, we define :

$$
\rho_{j}(f)=\frac{1}{p_{j}} \int_{\mathbb{R}^{d}} \frac{E_{A}^{0, x}\left(\sigma_{0, x}\right) f_{j}(x)}{r_{j}(x)} \lambda(x) d x \quad \text { and } \quad \rho(f)=\sup _{j} \rho_{j}(f) .
$$

$\mathcal{F}$ is a convex closed set and $f \rightarrow \rho(f)$ is a convex function, thus the minimum of Equation (15) is reached. In this section, we take interest to the optimal subset of $\mathcal{F}$ defined as :

$$
\mathcal{F}^{*}=\{f \in \mathcal{F}: \rho(f)=\rho\} .
$$

The extremal points of the convex set $\mathcal{F}$ are the measurable functions such that $f_{j}(x)=$ $\mathbb{1}\left(x \in V_{j}\right)$, for a Borel set $V_{j}$. This class of function is called tessellation. A tessellation is a partition of the space : each point $x \in \mathbb{R}^{d}$ is affiliated to a unique base station $j$.

We know that there is a mapping from an $\left(f_{j}\right)_{j}$ to a stable policy $\pi$. Therefore, in Equation (15) we are looking for a policy scheme which maximizes the intensity of arrival. The policy scheme which corresponds to a tessellation is a cellular type policy : a customer is served only by one base station.

Proposition 3. If $\rho$ is finite there is a $f$ such that :

$$
\forall j, \quad \rho_{j}(f)=\rho .
$$

If there is a finite number of server stations, all $f \in \mathcal{F}^{*}$ satisfy the above equation.

Proof. Let $f \in \mathcal{F}$ and suppose for example, $\rho_{1}(f)<\rho_{2}(f)$, since, $\rho_{2}(f)>0, f_{2}$ is not a.e. equal to 0 . Thus, there exists a measurable non negative function $x \mapsto \epsilon(x)$ such that $f_{2}^{\epsilon}(x)=f_{2}(x)-\epsilon(x) \geq 0, f_{1}^{\epsilon}(x)=f_{1}(x)+\epsilon(x) \leq 1$ and $\rho_{2}(\epsilon)>0$. Let $f_{j}^{\epsilon}(x)=f_{j}(x)$, for $j \notin\{1,2\} . f^{\epsilon} \in \mathcal{F}$ and we have $\rho_{1}\left(f^{\epsilon}\right)=\rho_{1}(f)+\rho_{1}(\epsilon)$ and $\rho_{2}\left(f^{\epsilon}\right)=\rho_{2}(f)-\rho_{2}(\epsilon)$. Thus for $\rho_{1}(\epsilon)$ small enough, $\sup _{j \in\{1,2\}} \rho_{j}\left(f^{\epsilon}\right)<\sup _{j \in\{1,2\}} \rho_{j}(f)$ and $\rho\left(f^{\epsilon}\right) \leq \rho(f)$.

Suppose now that $f \in \mathcal{F}^{*}$, then $f^{\epsilon}$ is also in $\mathcal{F}^{*}$. By iterating the construction above for all $j, j^{\prime}$, such that $\rho_{j^{\prime}}(f)<\rho_{j}(f)$, the proposition follows.

Proposition 3] as an intuitive meaning : for an optimal spatial allocation, the traffic load is the same on each server station. Along the same line, we can prove a more surprising result.

Definition 5. The processing rates are said to be singular if there exist $j$ and $k$ in $\mathcal{J}, a$ constant $C>0$ and a Borel set $A$ of positive Lebesgue measure such that :

$$
\forall x \in A, r_{j}(x)=C r_{k}(x) .
$$

Proposition 4. Suppose $\rho$ is finite. If the processing rates are not singular, then there is an $f \in \mathcal{F}^{*}$ which is a tessellation.

If there is finite number of base stations, all $f \in \mathcal{F}^{*}$ are tessellations. 
This proposition gives a counter-intuitive result : the server stations do not need to share the jobs to reach the stability region. However, note that all the difficulty is to find the optimal tessellation which reaches the optimum. This result is not very surprising from the point of view of convex optimization : it only asserts that the extremum is reached at an extremal point.

Definition [5] is purely technical and does not relies on a natural assumption on the processing rates. In the wireless scenario (Example 1.4) and $r_{j}(x)=f(x) l\left(\left|Y_{j}-x\right|\right)$, if $Y_{j} \neq Y_{k}$ for all $j, k \in \mathcal{J}$ and $l$ is a strictly convex mapping then the processing rates are non-singular.

Proof. We consider the $f \in \mathcal{F}^{*}$ given by proposition [3] Let $E=f_{1}(] 0,1[)^{-1} \cap f_{2}(] 0,1[)^{-1}$. In this proof, $\mu$ will denote the Lebesgue measure. We want to show that $\mu(E)=0$.

Suppose instead that $\mu(E)>0$, we can suppose without loss of generality that $\mu(E)<$ $+\infty$. Let $A, B$ be disjoint compact sets of positive Lebesgue measure included in $E$, these sets exist in view of Theorem 2.14 in Rudin [10] (Riesz Representation Theorem). We consider the mapping $\phi(x)=\mathbb{1}(x \in A)-\nu \mathbb{1}(x \in B), \nu>0$.

Let $f_{1}^{\epsilon}(x)=f_{1}(x)+\epsilon \phi(x), f_{2}^{\epsilon}(x)=f_{2}(x)-\epsilon \phi(x)$ and $f_{i}^{\epsilon}(x)=f_{i}(x)$ for $i \notin\{1,2\}$. If $\epsilon>0$ is small enough, $f^{\epsilon}$ and $f^{-\epsilon}$ are in $\mathcal{F}$ and for $i \in\{1,2\}$ :

$$
\rho_{i}\left(f^{ \pm \epsilon}\right)=\rho_{i}(f) \pm \epsilon \rho_{i}(\phi)=\rho \pm \epsilon \rho_{i}\left(\mathbb{1}_{A}\right) \mp \nu \epsilon \rho_{i}\left(\mathbb{1}_{B}\right)
$$

Since $f \in \mathcal{F}^{*}, \max \left(\rho_{1}\left(f^{ \pm \epsilon}\right), \rho_{2}\left(f^{ \pm \epsilon}\right)\right) \geq \rho$ and we deduce that $\operatorname{sign}\left(\rho_{1}(\phi)\right)=\operatorname{sign}\left(\rho_{2}(\phi)\right)$, where sign is the sign function $\left(\operatorname{sign}(0)=0\right.$, and for $\left.x \neq 0, \operatorname{sign}(x)=\frac{x}{|x|}\right)$. It follows that for all real $\nu, \rho_{1}\left(\mathbb{1}_{A}\right)-\nu \rho_{1}\left(\mathbb{1}_{B}\right)$ and $\rho_{2}\left(\mathbb{1}_{A}\right)-\nu \rho_{2}\left(\mathbb{1}_{B}\right)$ have the same sign. Therefore the vector $\left(\rho_{1}\left(\mathbb{1}_{A}\right), \rho_{1}\left(\mathbb{1}_{B}\right)\right)$ and $\left(\rho_{2}\left(\mathbb{1}_{A}\right), \rho_{2}\left(\mathbb{1}_{B}\right)\right)$ are colinear : exists $C_{A, B}$ such that : $\rho_{1}\left(\mathbb{1}_{A}\right)=C_{A, B} \rho_{2}\left(\mathbb{1}_{A}\right): C_{A, B}$ cannot depend on $B$ and by symmetry does not depend neither on $A$. Thus, exists $C>0$ such that :

$$
\rho_{1}\left(\mathbb{1}_{A}\right)=C \rho_{2}\left(\mathbb{1}_{A}\right) .
$$

This last equality has been proved for any compact set included in $E$. From Theorem 2.14 in [10, it can be extended to any Borel set included in $E$. Thus, for all $A$ included $E$, such that $\left.\mu(A)>0, \frac{1}{\mu(A)} \int_{A} \frac{E_{A}^{0, x}\left(\sigma_{0, x}\right)}{p_{1} r_{1}(x)}-C \frac{E_{A}^{0, x}\left(\sigma_{0, x}\right)}{p_{2} r_{2}(x)}\right) d x=0$. We can apply Theorem 1.40 of [10] and conclude that a.e. in $E$ :

$$
C^{\prime} r_{1}(x)=r_{2}(x) \text {. }
$$

This contradicts our hypothesis on the processing rates. Therefore $\mu(E)=0$. and we have proved that there exists an $f$ in $\mathcal{F}^{*}$ such that a.e. $f_{j}(x)=\mathbb{1}\left(x \in V_{j}\right)$ and $V_{j} \cap V_{k}$ is a set of measure 0 . Since for two functions equal a.e., $\rho_{j}(f)$ has the same value, $\left(\mathbb{1}\left(V_{j}\right)\right)_{j \in \mathbb{N}}$ is a tessellation in $\mathcal{F}^{*}$.

$\mathrm{RR} \mathrm{n}^{\circ} 5305$ 


\section{Cellular Policies}

Definition 6. Let $\left\{V_{j}\right\}, j \in \mathcal{J}$, be a tessellation, a cellular policy with cells $\left\{V_{j}\right\}_{j}$ is a policy scheme satisfying for all $j$ :

$$
\forall m \in \mathcal{M}, \quad \pi_{j}(m)\left(\mathbb{R}^{d} \backslash V_{j}\right)=0 .
$$

We have seen in Proposition 4 that cellular policies reach the stability region under some assumptions. In this section, we focus on this subclass of policies.

For a cellular policy, the server stations are not sharing the jobs which are divided among them. We say that a cellular policy is work-conserving if $m\left(V_{j}\right)>0$ implies $\pi_{j}(m)\left(V_{j}\right)=1$.

Proposition 5. Let $\left\{V_{j}\right\}, j \in \mathcal{J}$, be a tessellation with bounded sets, any work conserving cellular policy with cells $\left\{V_{j}\right\}_{j}$ is stable if:

$$
\forall j, \quad \int_{V_{j}} \frac{E_{A}^{0, x}\left(\sigma_{0, x}\right)}{r_{j}(x)} \lambda(d x)<p_{j} .
$$

If there is a $j$ such that : $\int_{V_{j}} \frac{E_{A}^{0, x}\left(\sigma_{0, x}\right)}{r_{j}(x)} \lambda(d x)>p_{j}$ then any cellular policy with cells $\left\{V_{j}\right\}_{j}$ is unstable.

This proposition is along the line of the result on single server queue which asserts that the stability does not depend on the discipline, provided it is work-conserving.

Since there is no interactions between server stations when a cellular policy is enforced, to prove the proposition, it is sufficient to prove the following result :

Suppose that the intensity measure $\lambda(d x)$ is finite and that there is a unique server. Then if $A \in \mathcal{N}^{s}$, any work conserving policy is stable, if $A \notin \overline{\mathcal{N}}^{s}$ any working policy is unstable.

This result on multi-class queues is mainly known. A proof is given in Appendix.

\section{Conclusion}

In this paper, we have derived some basic results on a spatial queuing system. An important aspect of this model is that the workload is an atomic measure with an infinite total mass and not a vector in $\mathbb{R}_{+}^{n}$. Another feature is the infinite number of server stations but this was more easy to handle.

With stronger hypothesis on the arrival point process $A$, it could be possible to derive more precise results on the stability and on the law of the stationary workload measure.

As already pointed out, Theorem 1 does not provide an interesting stationary policy. It would be interesting to find other types of policies which are stable when the arrival point process is in $\mathcal{N}^{s}$. In this scope, deriving the stability region of cone policies described in example 4.2 is a challenging work. 


\section{Appendix : Property of the Stability Set}

In this section, we find some properties on the set $\mathcal{N}^{s}$ as it is defined in Section 1.5 $\mathbb{N}$.

To simplify notations, $\nu_{j}(d x)$ will denote the measure $\frac{E_{A}^{0, x}\left(\sigma_{0, x}\right)}{r_{j}(x)} \lambda(d x)$ and $\mathcal{J}$ is set to be

For $x \in \mathbb{R}^{d}$, let $\mathcal{J}_{x}=\left\{j: f_{j}(x)>0\right\}$ and for a set $B, \mathcal{J}_{B}=\cup_{x \in B} \mathcal{J}_{x}$.

Proposition 6. If $\mathcal{N}^{s}$ is not empty, there is a $f \in \mathcal{F}$ such that for all $j, x \mapsto f_{j}(x)$ is continuous and for all bounded set $B,\left|\mathcal{J}_{B}\right|$ is finite.

Proof. Let $f \in \mathcal{F}$ such that $\forall j, \rho_{j}=\int_{\mathbb{R}^{d}} f_{j}(x) \nu_{j}(d x)<1$. Let $G$ be a open bounded set.

For all $j$, from Lusin's Theorem (see for example [10]) there exists a sequence of continuous functions on $G, g_{j}^{n}(\bullet)$, such that $\nu_{j}(d x)$-a.e., $0 \leq g_{j}^{n}(x) \leq f_{j}(x)$ and $\lim _{n} \int_{G} g_{j}^{n}(x) \nu_{j}(d x)=$ $\int_{G} f_{j}(x) \nu_{j}(d x)$.

We have $\sum_{j} g_{j}^{n}(x) \leq 1=\sum_{j} f_{j}(x)$. Let $\epsilon^{n}(x)=1-\sum_{j \neq 1} g_{j}^{n}(x)$. We define

$$
f_{j}^{n}(x)=\left\{\begin{array}{lll}
g_{j}^{n}(x)+\mathbb{1}_{j=1} \epsilon^{n}(x) & \text { if } & x \in G, \\
f_{j}(x) & \text { if } & x \notin G,
\end{array}\right.
$$

We check that $f^{n}$ is in $\mathcal{F}$ and by the dominated convergence theorem, for $n$ large enough $: \forall j, \int_{\mathbb{R}^{d}} f_{j}^{n}(x) \nu_{j}(d x)<1$.

By iterating this construction for a set of open sets covering the $\mathbb{R}^{d}$, we deduce that there exists and $f \in \mathcal{F}$, such that $\forall j, \rho_{j}=\int_{\mathbb{R}^{d}} f_{j}(x) \nu_{j}(d x)<1$ and $f_{j}$ continuous.

Now we turn to the second part of the proposition. Let $K$ be a compact subset, $f_{j}$ as above and $\max \left(\rho_{j}, \frac{1}{2}\right)<\rho_{j}^{\prime}<1$. We define $g_{j}(x)=\frac{f_{j}(x)}{\rho_{j}^{\prime}}$.

Then : $\int_{\mathbb{R}^{d}} g_{j}(x) \nu_{j}(d x)=\frac{\rho_{j}}{\rho_{j}^{\prime}}<1$ and $\sum_{j} g_{j}(x)>1$. For all $x$, there exists $j_{x}$ such that $\sum_{j=1}^{j_{x}} g_{j}(x)>1$. By continuity, since $K$ is compact, $j_{K}=\sup _{x \in K} j_{x}$ is finite.

It is immediate to check that $\tilde{f}_{j}(x)=\frac{g_{j}(x) \mathbf{1}_{j \leq j_{x}}}{\sum_{j \leq j_{x}} g_{j}(x)}$ has all the required properties.

\section{Appendix : Vague Convergence in $\mathcal{M}$}

In this paragraph, we give some technical results used in Section 4

The following lemma is an adaptation of Theorem 5.2 of Billingsley [4 to the vague topology.

Lemma 6. Let $m_{n}$ be a sequence in $\mathcal{M}$ converging for the vague topology toward $m$. Let $h$ be a bounded measurable function which $m(\operatorname{disc}(h))=0$ and $B$ a bounded Borel set of $\mathbb{R}^{d}$ with $m(\partial B)=0$, then : $\lim _{n} \int_{B} h(x) m_{n}(d x)=\int_{B} h(x) m(d x)$

Lemma 7. Let $C$ be a countable set of points in $\mathbb{R}^{d}$ and let $\mathcal{C}$ the set of bounded Borel sets of $\mathbb{R}^{d}$ with $C \cap \partial B=\emptyset$. Then :

- $\mathcal{C}$ is an algebra and the $\sigma$-algebra generated by $\mathcal{C}, \sigma(\mathcal{C})$ is the Borel $\sigma$-algebra $\mathcal{B}$. 
- From a measure $m$ defined on $\mathcal{C}$, there is a unique extension to $m$ on $\mathcal{B}$.

Proof. From the relations : $\partial(A \cap B) \subset \partial A \cap \partial B, \partial(A \cup B) \subset \partial A \cup \partial B$ and $\partial\left(A^{c}\right)=\partial A$. It follows that $\mathcal{C}$ is an algebra on the set of sets of $\mathbb{R}^{d}$.

$\mathcal{B}$ is the $\sigma$-algebra of the algebra generated by the open rectangles of $\mathbb{R}^{d}$. To prove the first assertion of our lemma, it suffices to prove that the rectangle $] 0,1\left[^{d}\right.$ can be written as $\cup_{n \in \mathbb{N}} B_{n}$, where $B_{1} \subset B_{2} \ldots \subset B_{n} \in \mathcal{C}$. To this end, consider the rectangle $\left.R_{\epsilon}=\right] \epsilon, 1-\epsilon{ }^{d}$, with $0<\epsilon<\frac{1}{2}$. If $\epsilon \neq \epsilon^{\prime}, \partial R_{\epsilon} \cap \partial R_{\epsilon^{\prime}}=\emptyset$. Since $C$ is countable there can only be a countable set of $\epsilon$ such that $C \cap \partial R_{\epsilon} \neq \emptyset$. In particular, there exists an increasing sequence $\epsilon_{n}$ such that $C \cap \partial R_{\epsilon_{n}}=\emptyset$. We have proved the first statement of the lemma.

The second assertion is the Caratheodory Extension Theorem.

\section{Appendix : Spatial queuing system with one server}

When there is only one server in the system, Theorem 1 can be made more precise. The system reduces to a multi-class queue. The condition $\lambda \in \mathcal{N}^{s}$ can be restated as :

$$
\int_{\mathbb{R}^{d}} \frac{E_{A}^{0, x}\left(\sigma_{0, x}\right)}{r(x)} \lambda(d x)<p
$$

where $r$ is the processing rate for the server providing service for a user located at $x$ and $p$ is the expectation of the available processing power.

We have the following proposition :

Proposition 7. Suppose that the intensity measure $\lambda(d x)$ is finite and that there is a unique server. Then if $A \in \mathcal{N}^{s}$, any work conserving policy is stable.

Proof. We will only give a sketch of the proof in three steps. We will suppose that $\epsilon(t)=1$ (hence $p=1$ ). The proof in the general case follows with obvious modifications.

Step 1. Since $\lambda$ is a finite Radon measure, $N=\sum_{n} \delta_{T_{n}, \frac{\sigma_{n}}{r\left(X_{n}\right)}}$ is a simple marked point process on $\mathbb{R}$ with finite intensity $E(N[0,1])=\int_{\mathbb{R}^{d}} \lambda(d x)$.

For a given work conserving policy $\pi$, define $Y_{t}=\int_{\mathbb{R}^{d}} \frac{W_{t}(d x)}{r(x)}$. From Equation (6), we deduce that for $t \in\left[T_{n}, T_{n+1}\right)$ :

$$
Y_{t}=\left(Y\left(T_{n}-\right)+\frac{\sigma_{n}}{r\left(X_{n}\right)}+\left(t-T_{n}\right)\right)^{+} .
$$

Therefore, $Y_{t}$ does not depend on the policy and $Y_{t}$ is the usual workload for $G / G / 1$ queue (in particular $W_{t}\left(\mathbb{R}^{d}\right)$ is equal to zero at the same time for any work conserving policy). The workload for this queue is equal to $\int_{\mathbb{R}^{d}} \lambda(d x) E_{N}^{0}\left(\frac{\sigma_{0}}{r\left(X_{0}\right)}\right)=\int_{\mathbb{R}^{d}} \frac{E_{A}^{0, x}\left(\sigma_{0, x}\right)}{r(x)} \lambda(d x)<1$.

Similarly, by denoting $W_{n}=W_{T_{n}-}$, it appears that $\left(W_{n}\right), n \geq 0$, is generated by a stochastic recurrence, see [6], 2] (indeed we may write $W_{n+1}=h\left(W_{n}, \xi_{n}\right)$, for a suitable measurable mapping $h$ and a driving sequence $\left(\xi_{n}\right), n \in \mathbb{N}$, compatible with the flow $\theta$ ).

INRIA 
Step 2. Let $\mathcal{M}_{f}$ be the set of atomic measures with a finite set of atoms on $\mathbb{R}^{d}$. If $W_{0} \in \mathcal{M}_{f}$ then a.s., $W_{t} \in \mathcal{M}_{f}$ for $t \geq 0$.

Now, we define the following policy on $\mathcal{M}_{f}$ :

$$
\pi^{-}:\left\{\begin{aligned}
\mathcal{M}_{f} & \rightarrow \mathcal{M} \\
m & \mapsto\left\{\begin{array}{lll}
\delta_{x^{*}} & \text { if } & m \neq 0 \\
0 & \text { if } & m=0 .
\end{array}\right.
\end{aligned}\right.
$$

where $x^{*}=\arg \max \{x: r(x) \mathbb{1}(m(\{x\})>0)\}$. If multiple choices of $x^{*}$ are possible, choose the first in the lexicographic order.

$\pi^{-}$is the work conserving policy which dedicates all the processing power to the slowest customer. For $\pi^{-}, m \rightarrow W_{t}^{m}$ is non-increasing : that is, $m^{\prime} \succeq m$ implies for all $t \geq 0$, $W_{t}^{m^{\prime}} \succeq W_{t}^{m}$. To prove this last assertion, remark that for $t \in\left[0, T_{1}\right)$ and $t$ small enough :

$$
W_{t}^{m^{\prime}}=m^{\prime}-\operatorname{tr}\left(x^{*}\left(m^{\prime}\right)\right) \delta_{x^{*}\left(m^{\prime}\right)}
$$

and

$$
W_{t}^{m}=m-\operatorname{tr}\left(x^{*}(m)\right) \delta_{x^{*}(m)} .
$$

If $m\left(\left\{x^{*}\left(m^{\prime}\right)\right\}\right)>0$ then $W_{t}^{m^{\prime}}-W_{t}^{m}=m^{\prime}-m \succeq 0$. If $m\left(\left\{x^{*}\left(m^{\prime}\right)\right\}\right)=0$ then since $m^{\prime}\left(\left\{x^{*}(m)\right\}\right)>0$, we have still $W_{t}^{m^{\prime}}-W_{t}^{m} \succeq 0$.

Let $M_{t}^{-}$be the Loynes'sequence for policy $\pi^{-}$(a.s., $M_{t}^{-} \in \mathcal{M}_{f}$ ). Let $0 \leq s \leq t$, since $0=W_{0}^{0} \circ \theta_{-s} \preceq W_{t-s}^{0} \circ \theta_{-t}$, from the monotonicity of $m \mapsto W_{s}^{m}$, we deduce that : $M_{s}^{-}=W_{s}^{0} \circ \theta_{-s} \preceq W_{s}^{W_{t-s}^{0} \circ \theta_{-t}} \circ \theta_{-s}=M_{t}^{-}$.

Thus $\left(M_{t}^{-}\right), t \in \mathbb{R}^{+}$, is a non-decreasing sequence and for all Borel set $B, M_{t}^{-}(B) \leq$ $M_{t}^{-}\left(\mathbb{R}^{d}\right) . M_{t}^{-}\left(\mathbb{R}^{d}\right)$ is the Loynes' sequence for the usual $G / G / 1$ queue (from Step 1 ). We deduce that $M_{t}^{-}$converges a.s. toward the Loynes' variable $M_{\infty}^{-}$(from Lemma 4 ).

Step 3. Consider now any work conserving policy $\pi$, we define similarly the Loynes' variable $M_{t}$ for policy $\pi$. As noticed above, $\pi^{-}$is the slowest policy and it follows :

$$
M_{t}\left(\mathbb{R}^{d}\right) \leq M_{t}^{-}\left(\mathbb{R}^{d}\right) \leq M_{\infty}^{-}\left(\mathbb{R}^{d}\right)
$$

Consider the event $A=\left\{M_{T_{n}}^{-}=0\right\}$. This event is a renovating event for $M_{T_{n}}$ and since the workload of the $G / G / 1$ queue is strictly less than $1, P_{N}^{0}(A) \geq P_{N}^{0}\left(M_{\infty}^{-}\left(\mathbb{R}^{d}\right)=0\right)>0$. From Theorem 2.5.3 and Property 2.5.5 of [2], we deduce that $M_{t}$ converges to a stationary solution $M_{\infty}$ and that $M_{t}$ couples with $M_{\infty}$ (in the strong backward sense).

Restating Property 2.4.1 of [2, we can also prove that from any finite initial condition $m, W_{t}^{m}$ couples with $M_{\infty} \circ \theta_{t}$ (as $t$ tends toward $+\infty$ ).

RR $n^{\circ} 5305$ 


\section{References}

[1] Armony, M. and Bambos, N. (2003). Queueing dynamics and maximal throughput scheduling in switched processing systems. Queueing Syst. 44, 209-252.

[2] Baccelli, F. And Bremaud, P. (2003). Elements of Queuing Theory second ed. Applications of Mathematics. Springer, Berlin.

[3] Bambos, N. and Michailidis, G. (2004). Queueing and scheduling in random environments. Adv. in Appl. Probab 36, 293-317.

[4] Billingsley, P. (1968). Convergence of Probability Measures. Wiley Series in Probability and Mathematical Statistics. Wiley.

[5] Bordenave, C. (2004). Stability properties of data flows on a cdma network in macrodiversity. INRIA research report $\mathrm{RR}-5257$.

[6] Borovkov, A. (1994). Ergodicity and Stability of Stochastic Processes. Wiley Series in Probability and statistics. Wiley.

[7] Daley, D. and Vere-Jones, D. (1988). An introduction to the Theory of Point Processes. Springer Series in Statistics. Springer-Verlag, New-York.

[8] K. Wasserman, G. Michailidis, N. B. (2001). Differntiated processors' scheduling on heterogeneous task flows. Technical Report SU-Netlab-2001-12/1 Engineering Library, Stanford University.

[9] Lindvall, T. (1992). Lectures on the Coupling Method. Wiley.

[10] Rudin, W. (1974). Real and complex Analysis second edition ed. McGraw-Hill Series in Higher Mathematics. McGraw-HillBook Co.

[11] Tassiulas, L. and Eprhemides, A. (1992). Stability properties of constrained queueing systems and scheduling policies for maximum troughput in multihop radio networks. IEEE Transactions on Automatic Control 37, 1936-1948. 


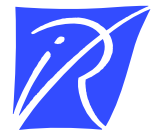

Unité de recherche INRIA Rocquencourt Domaine de Voluceau - Rocquencourt - BP 105 - 78153 Le Chesnay Cedex (France)

Unité de recherche INRIA Lorraine : LORIA, Technopôle de Nancy-Brabois - Campus scientifique 615, rue du Jardin Botanique - BP 101 - 54602 Villers-lès-Nancy Cedex (France)

Unité de recherche INRIA Rennes : IRISA, Campus universitaire de Beaulieu - 35042 Rennes Cedex (France)

Unité de recherche INRIA Rhône-Alpes : 655, avenue de l'Europe - 38330 Montbonnot-St-Martin (France)

Unité de recherche INRIA Sophia Antipolis : 2004, route des Lucioles - BP 93 - 06902 Sophia Antipolis Cedex (France) 